\title{
TWO EXACT SEQUENCES IN RATIONAL HOMOTOPY THEORY RELATING CUP PRODUCTS AND COMMUTATORS
}

\author{
LARRY A. LAMBE
}

\begin{abstract}
Let $X$ be an $(n-1)$-connected topological space of finite rational type (i.e. $H_{n}(X ; Q)$ is finite dimensional over $Q$ for all $n$ ). Sullivan's notion of minimal model is used to derive two exact sequences involving the kernel of the cup product operation in dimension $n$ and Whitehead products. The first of these generalizes both a theorem of John C. Wood [JCW] and a theorem of Dennis Sullivan [DS] and states that the kernel of the cup product map $H^{1}(X) \wedge H^{1}(X) \rightarrow H^{2}(X)$ is rationally the dual of the second factor of the lower central series of the fundamental group. Other examples are given in the last section.
\end{abstract}

1. Statement of the theorem. Let $X$ be an $(n-1)$-connected topological space and let $H^{n}(X)$ denote its $n$th singular rational cohomology group. The object of this paper is to derive the following two exact sequences:

THEOREM 1. The sequences

$$
\begin{gathered}
0 \rightarrow\left(\Gamma_{2} / \Gamma_{3} \otimes Q\right)^{*} \stackrel{d}{\rightarrow} H^{1} \wedge H^{1} \stackrel{\cup}{\rightarrow} H^{2} \quad \text { for } n=1, \\
0 \rightarrow H^{2 n-1} \stackrel{-h}{\rightarrow}\left(\pi_{2 n-1} \otimes Q\right)^{*} \stackrel{g}{\rightarrow} H^{n} \wedge H^{n} \stackrel{\cup}{\rightarrow} H^{2 n} \quad \text { for } n>1
\end{gathered}
$$

are exact, where $\pi_{i}=\pi_{i}(X)$, and $\Gamma_{i}$ is the ith term in the lower central series of $\pi_{1}$, $H^{i}=H^{i}(X ; Q), d$ is dual to the commutator map [ ]: $\pi_{1} / \Gamma_{2} \wedge \pi_{1} / \Gamma_{2} \rightarrow \Gamma_{2} / \Gamma_{3}, h$ is dual to the Hurewicz map $\mathscr{H}, g$ is dual to the composition of $\mathscr{H} \otimes \mathscr{H}$ and the Whitehead product [ ]: $\pi_{n} \otimes \pi_{n} \rightarrow \pi_{2 n-1}$ and $\wedge$ denotes exterior product if $n$ is odd and symmetric tensor product if $n$ is even.

The sequence (1.1) above is stated for the special case of compact oriented 3-manifolds in [DS] without proof. The case $\Gamma_{2}=0$ (i.e., $\pi_{1}$ abelian) of (1.1) gives Corollary 2 of [JCW]. Note that (1.1) applies to groups $G$ and group cohomology if $X=K(G ; 1)$ is used. The author is grateful to A. Libgober for inquiring about the validity of (1.1) in its stated generality. We note that versions of these exact sequences may also be derived from known spectral sequences [BK, LS] (see also

Received by the editors July 22, 1983 and, in revised form, January 10, 1985.

1980 Mathematics Subject Classification. Primary 55P62, 55Q15, 55 N99.

Key words and phrases. de Rham complex, Hurewicz map, lower central series, 1-minimal model, minimal model. 
[HS, 4.14; DQ]). The approach in this paper was chosen because of its elementary nature, assuming some properties of minimal models.

2. Notation and review. In this paper all coefficients will be in $Q$. A differential graded augmented algebra (DGA-alg) is a graded vector space $A=\oplus_{n \geqslant 0} A^{n}$ over $Q$ with an associative, graded commutative multiplication $A^{n} \otimes A^{m} \rightarrow A^{n+m}$ and an augmentation $\varepsilon: A \rightarrow Q \rightarrow 0$ and a differential $d: A^{n} \rightarrow A^{n+1}$ which is a derivation of $A$ [Mac]. All DGA-algs will be of finite $Q$ type. Given a degree 0 cochain map $f$ : $C \rightarrow D$ the "mapping cylinder" construction is the DGA-alg $M_{f}$ with $\left(M_{f}\right)^{n}=C^{n}$ $\oplus D^{n-1}, d(x, y)=(d(x), f(x)-d(y))$.

From the short exact sequence $0 \rightarrow D^{n-1} \rightarrow\left(M_{f}\right)^{n} \rightarrow C^{n} \rightarrow 0, i(y)=(0,-y)$, $q(x, y)=x$, we obtain the long exact sequence

$$
\cdots \rightarrow H^{n-1}(C) \rightarrow H^{n-1}(D) \rightarrow H^{n}(f) \rightarrow H^{n}(C) \rightarrow \cdots,
$$

where $H^{*}(f)=H^{*}\left(M_{f}\right)$. Note that the connecting homomorphism $\delta^{*}$ satisfies $\delta^{*}=-f^{*}$. If $V$ is a graded vector space then $\Lambda(V)$ will denote the free graded commutative augmented algebra generated by $V$.

2.2. 1-minimal model. For a DGA-alg $A$ such that $H^{0}(A)=Q$ there is an associated DGA-alg $M_{1}$ which possesses a filtration $M_{1, n} \leqslant M_{1, n+1}, n \geqslant 0$, with $M_{1,0}=Q, M_{1}=\bigcup_{n \geqslant 0} M_{1, n}$, and a degree 0 cochain map $p: M_{1} \rightarrow A$ that induces an isomorphism on $H^{1}$ and a monomorphism on $H^{2}$. Inductively one has:

(i) $V_{1}=H^{1}(A)$ and $M_{1,1}=\Lambda\left(V_{1}\right)$ with trivial differential.

(ii) $p_{1}: M_{1,1} \rightarrow A$ is given by a choice of algebra generators: $p_{1}(\operatorname{cls}(a))=a$.

(iii) $M_{1, n+1}=M_{1, n} \otimes \Lambda\left(V_{n+1}\right)$, where $V_{n+1}=H^{2}\left(p_{n}\right)$.

(iv) The differential on $M_{1, n+1}$ is given by choosing a splitting of the natural quotient map $Z^{2}\left(p_{n}\right) \rightarrow H^{2}\left(p_{n}\right)$, say $s(v)=\left(m_{v}, a_{v}\right)$ and extending $\left.d\right|_{M_{1, n}}=$ old $d$, $d(v)=m_{v}, v \in V_{n+1}$, as a derivation.

(v) $p_{n+1}: M_{1, n+1} \rightarrow A$ is induced by extending $\left.p_{n+1}\right|_{M_{1, n}}=p_{n}, p_{n+1}(v)=a_{v}$, $v \in V_{n+1}$.

Note. Elements of $V_{n}, n \geqslant 1$, are to be considered as having degree 1. $M_{1}$ is called the 1-minimal model of $A$. It is unique up to isomorphism.

2.3. Minimal model. For a DGA-alg $A$ such that $H^{0}(A)=Q$, and $H^{1}(A)=0$ there is an associated DGA-alg $M$ which possesses a filtration $M_{n} \leqslant M_{n+1}, n \geqslant 0$, with $M_{1}=Q, \quad M=\cup_{n \geqslant 0} M_{n}$, and a degree 0 cochain map $p: M \rightarrow A$ which induces an isomorphism in cohomology. Inductively one has:

(i) $M_{0}=M_{1}=Q, V_{2}=H^{2}(A)$ and $M_{2}=\Lambda\left(V_{2}\right)$ with trivial differential.

(ii) $p_{2}: M_{2} \rightarrow A$ is given by a choice of algebra generators.

(iii) $M_{n+1}=M_{n} \otimes \Lambda\left(V_{n+1}\right)$, where $V_{n+1}=H^{n+2}\left(p_{n}\right)$.

(iv) $\left.d\right|_{M_{n}}=$ old $d, d(v)=m_{v}$, where $s(v)=\left(m_{v}, a_{v}\right)$, as before.

(v) $p_{n+1}: M_{n+1} \rightarrow A$ is induced by extending $\left.p_{n+1}\right|_{M_{n}}=p_{n}, p_{n+1}(v)=a_{v}$.

Note. Elements of $V_{n}, n \geqslant 2$, are to be considered as having degree $n$. $M$ is called the minimal model of $A$. It is unique up to isomorphism. The constructions above are in [GM]. 
2.4. REMARK. For a DGA-alg such that $H^{0}(A)=Q$ there is a notion of minimal model which combines the notions of 1 -minimal model and minimal model [DS2, BG]. Using this one can obtain a "twisted version" of (1.1) and (1.2) combined for "nilpotent spaces", i.e. spaces for which $\pi_{1}$ is nilpotent and acts nilpotently on $\pi_{n}$ for all $n$.

3. Proof of Theorem 1. In this section let $A=A^{*}(X)$ be the rational de Rham complex of $X$.

3.1. Let $V_{n}$ be as in 2.2 and $W_{n}=V_{1} \oplus V_{2} \oplus \cdots \oplus V_{n}$. We have that $W_{n} \rightarrow W_{n-1}$ $\wedge W_{n-1} \hookrightarrow W_{n} \wedge W_{n}$ induces a Lie algebra structure: $d^{*}: W_{n}^{*} \wedge W_{n}^{*} \rightarrow W_{n}^{*}$. The main theorem on 1-minimal models states that $W_{n}^{*}=\mathscr{L}\left(\pi_{1}(X) / \Gamma_{n+1}\right)$ (the Mal'cev Lie algebra of the nilpotent group $\left.\pi_{1} / \Gamma_{n+1}\right)$. Thus we have $\mathscr{L}\left(\pi_{1} / \Gamma_{3}\right)=W_{2}{ }^{*}=V_{1}^{*}$ $\oplus V_{2}^{*}$ additively.

The nontrivial part of the Lie bracket map is [, ]: $V_{1}^{*} \wedge V_{1}^{*} \rightarrow V_{2}^{*}$ and the nilpotency class of $\pi_{1} / \Gamma_{3}$ is $\leqslant 2$ so its Mal'cev Lie algebra is the same as the graded Lie algebra associated to its lower central series (see [LP, 7] for an example of a nilpotent group such that $\mathscr{L}(G) \neq \operatorname{gr}(G))$. Since $V_{1}^{*}=\left(\pi_{1} / \Gamma_{2} \otimes Q\right)^{*}$ it follows that $V_{2}^{*}=\left(\Gamma_{2} / \Gamma_{3} \otimes Q\right)^{*}$ and that $[$,$] is given by group commutator. Now in this case$ the long exact sequence of the mapping cylinder (2.1) becomes

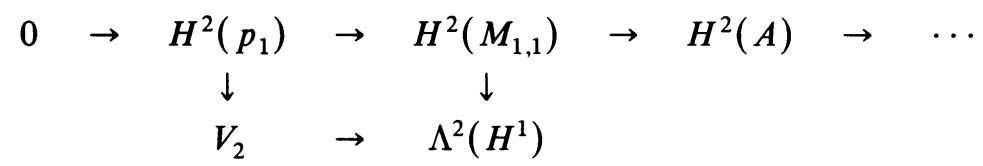

and the map $d^{*}$ is easily identified as [, ]. With these identifications the exact sequence (1.1) is just (3.2).

3.3. Let $V_{n}$ be as in 2.3 and $V_{*}=\oplus_{n \geqslant 2} V_{n}$ and let $M^{+}=\oplus_{j>0} M^{j}$. The main theorem on minimal models states that for a 1-connected space $X, V_{n}^{*}=\pi_{n}$ and the differential from 2.2(iv) induces a map

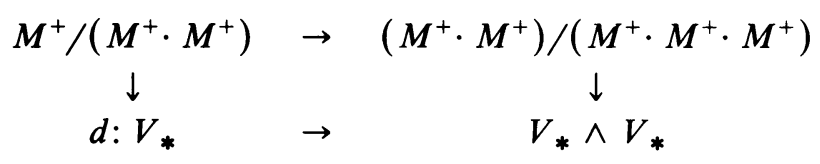

where $d$ is dual to the Whitehead product [ , ]: $\pi_{*} \wedge \pi_{*} \rightarrow \pi_{*}$ (a degree -1 map) [GM, AA, BG, DS2]. To obtain the exact sequence (1.2) we assume that $X$ is $(n-1)$-connected, $n>1$. We have

3.4. LeMma. (i) For $1 \leqslant k \leqslant n-2$

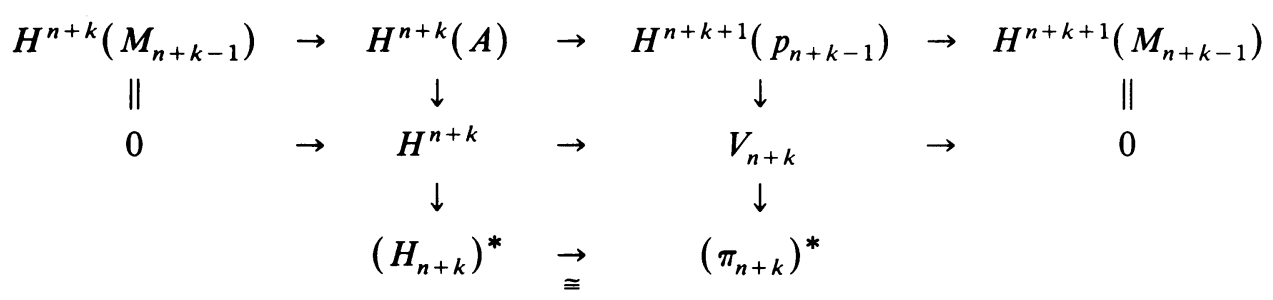

where the bottom arrow is dual to the Hurewicz map. 
(ii) For $k=n-1$

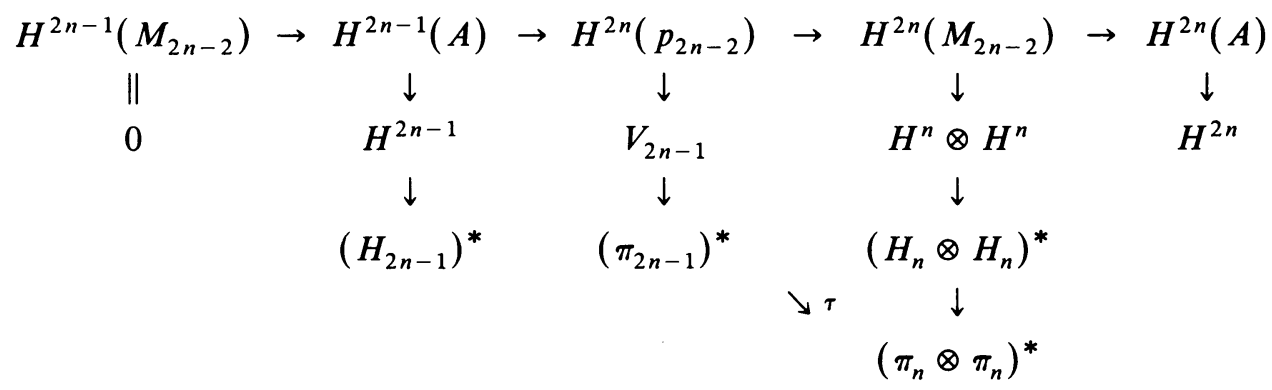

where the diagonal map is dual to the Whitehead product.

Proof. Using the minimal model construction 2.3 we have $M_{j}=Q, j<n$, and $M_{n}=\Lambda\left(V_{n}\right)=\Lambda\left(H^{n}\right)$ with $d=0$. Thus (2.1) becomes:

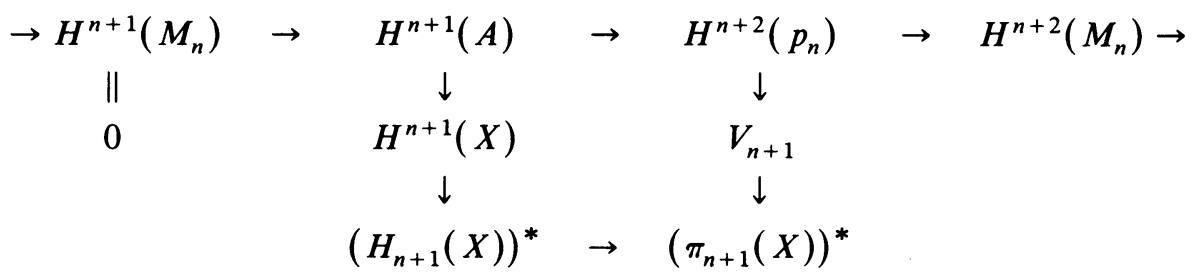

By the general theory, the vertical maps may be chosen so that the bottom map is dual to the Hurewicz map [GM]. By definition, $M_{n+1}=M_{n} \otimes \Lambda\left(V_{n+1}\right)$ and in this case we can choose a splitting $s: H^{n+2}\left(p_{n}\right) \rightarrow Z^{n+2}\left(p_{n}\right)$ such that $s(v)=\left(0, a_{v}\right)$ to get the differential. Thus $d(v)=0$ and $p_{n+1}(v)=a_{v}$. This is the first step in an induction which shows that for all $j$ such that $1 \leqslant j \leqslant n-2, M_{n+j}=\Lambda\left(V_{n} \oplus V_{n+1}\right.$ $\oplus \cdots \oplus V_{n+j}$ ) with trivial differential. This statement is equivalent to (i) (choosing the vertical isomorphisms correctly). It is now clear that (ii) is just the exact sequence (2.1) with the vertical maps coming from the general theory. For the diagonal map $\tau$ note that $q^{*}(v)=\operatorname{cls}\left(m_{v}\right)$ where we have chosen a splitting $s: H^{2 n}\left(p_{2 n-2}\right) \rightarrow$ $Z^{2 n}\left(p_{2 n-2}\right), s(v)=\left(m_{v}, a_{v}\right)$. Since $m_{v} \in Z^{2 n}\left(M_{2 n-2}\right)=\Lambda\left(V_{n} \oplus \cdots \oplus V_{2 n-2}\right)$, it can at most be quadratic; but we also have that $d(v)=m_{v}$. Since the Whitehead product is determined by the quadratic part of the differential [DS, AA] we are done.

4. Examples. In [DS] a special case of (1.1) is used to show that $S^{1} \times S^{1} \times S^{1}$ is not the link of an isolated singularity of a complex algebraic surface. It is noted in [JCW] that the case of $\pi_{1}$ abelian yields a result of $\mathrm{H}$. Hopf: If $\pi_{1}(X)$ is Abelian, then the first and second Betti numbers of $X$ satisfy $\beta_{1}\left(\beta_{1}-1\right) / 2 \leqslant \beta_{2}[\mathbf{H H}]$.

In the special case that $X=G / D$ is a nilmanifold, i.e. the homogeneous space determined by a nilpotent Lie group $G$ and a discrete uniform subgroup $D,(1.1)$ follows immediately from the well-known fact that if $\mathscr{L}$ is the rational graded Lie algebra associated to the lower central series of $D$, then the 1-minimal model of $X$ is just the Koszul complex of $\mathscr{L}$ [DS2, LP]. In this context, (1.1) leads to an explicit construction of Massey products [JPM] in terms of the commutator structure. 
4.1. Higher dimensions. In certain cases the present methods can be used to derive a generalization of the higher dimensional statements in [JCW, Theorem 1] by giving the kernel of the cup product in an explicit form. (1.2) is an example of this.

Note that (1.2) also contains the presumably well-known result that the $(2 n-1)$ st homotopy group of an $(n-1)$-connected $2 n$-manifold is the kernel of cup product in dimension $n$ rationally.

4.2. Final remarks. It should be noted that there are spaces of interest for which the theory fails. For example let $V^{n}$ be the algebraic variety defined by the equation $\sum_{i=1}^{n+1}\left(f_{q_{i}}\right)=P_{i}=0$, where $q_{1}=\prod_{i=1}^{n+1} p_{j} / p_{i}, 1 \leqslant i \leqslant n+1$, and $f_{q_{i}}$ denotes a generic form of degree $q_{i}$. It is shown in [AL] that $\pi_{n}\left(C P_{n+1}-\left(V^{n} \cup H\right)\right) \otimes Q \neq 0$, where $H$ is a generic hyperplane. For example, the space corresponding to the equation $\left(f_{21}\right)^{2}+\left(f_{14}\right)^{3}+\left(f_{6}\right)^{7}=0$ has $\pi_{1}=Z, H_{2}=0$. If the fundamental group acted nilpotently on higher homotopy the twisted theory mentioned in 2.4 would give that $\pi_{2}=0$.

Finally, the particular construction of the minimal model used in this paper was chosen to accomodate our special purpose. For other purposes other forms of the minimal model may be more useful. This is well illustrated in [HS].

\section{REFERENCES}

[AA] Peter Andrews and Martin Arkowitz, Sullivan's minimal models and higher order Whitehead products, Canad. J. Math. 30 (1978), 961-982.

[AC] C. Allday, Rational Whitehead products and a spectral sequence of Quillen, Pacific J. Math. 46 (1973), 305-330.

[AL] A. Libgober, Homotopy groups of complements to singular hypersurfaces, Univ. of Illinois at Chicago, preprint.

[BG] A. K. Bousfield and V. K. A. M. Gugenheim, On PL de Rham theory and rational homotopy type, Mem. Amer. Math. Soc. No. 179 (1976).

[BK] A. K. Bousfield and D. M. Kan, Pairings and products in the homotopy spectral sequence, Trans. Amer. Math. Soc. 177 (1973), 319-343.

[DQ] D. Quillen, Rational homotopy theory, Ann. of Math. (2) 90 (1969), 205-295.

[DS] Dennis Sullivan, On the intersection ring of compact three manifolds, Topology 14 (1975), 275-277.

[DS2] _ Infinitesimal computations in topology, Publ. Inst. Hautes Études Sci., no. 47, 1977, pp. 269-331.

[GM] Phillip A. Griffiths and John W. Morgan, Rational homotopy theory and differential forms, Progress in Math., vol. 16, Birkhauser, Boston, Mass., 1981.

[HH] H. Hopf, Fundamentalgruppe und Zweite Bettische Gruppe, Comment. Math. Helv. 14 (1942), 257-309.

[HS] Steven Halperin and James Stasheff, Obstructions to homotopy equivalences, Adv. in Math. (2) 32 (1979), 233-279.

[JCW] J. C. Wood, A theorem on injectivity of the cup product, Proc. Amer. Math. Soc. 37 (1973), $301-304$.

[JPM] J. P. May, Matric Massey products, J. Algebra 12 (1969), 533-568.

[LP] L. Lambe and S. Priddy, Cohomology of nilmanifolds and torsion-free nilpotent groups, Trans. Amer. Math. Soc. 273 (1982), 39-55.

[LS] L. Smith, Lectures on the Eilenberg-Moore spectral sequence, Lecture Notes in Math., vol. 134, Springer-Verlag, Berlin and New York, 1970.

[Mac] S. Mac Lane, Homology, Die Grundlehren der Math. Wissenschaften, Band 114, Springer-Verlag, New York, 1963.

Department of Mathematics, Statistics and Computer Science, University of Illinois at Chicago, Chicago, Illinois 60680

Current address: Department of Mathematics, North Carolina State University, Raleigh, North Carolina 27695 